\title{
ON THE VALIDATION OF VESTA 2.2.0 USING THE ARIANE-GU3 SAMPLE
}

\author{
R. Ichou ${ }^{1}$, B. Dechenaux ${ }^{1}$ \\ ${ }^{1}$ IRSN, PSN-EXP/SNC/LN \\ 31 avenue de la Division Leclerc, Fontenay-aux-Roses, France \\ raphaelle.ichou@irsn.fr
}

\begin{abstract}
The validation of the VESTA 2.2.0 Monte Carlo depletion code has been initiated using the Spent Fuel Isotopic Composition Database (SFCOMPO). The work presented in this paper is limited to one fuel sample, the GU3 PWR-UOX sample from the ARIANE program, which

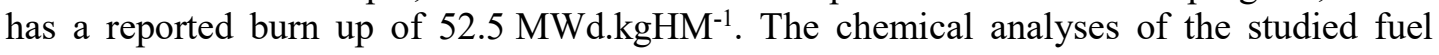
sample were performed by 2 independent laboratories at the end of irradiation and cooling time. US and European evaluated nuclear data libraries, namely ENDF/B-VII.1 and JEFF3.2, but also the more recent ENDF/B-VIII.0 and JEFF-3.3 are used for the VESTA 2.2.0 calculations. The isotopic concentration results are compared to experimental data and the $\mathrm{C} / \mathrm{E}$ agreement is analyzed in the light of the previous VESTA 2.1.5 validation results obtained using ENDF/B-VII.0 and JEFF-3.1 nuclear data libraries.
\end{abstract}

KEYWORDS: VESTA 2.2.0 validation, ARIANE GU3, SFCOMPO

\section{INTRODUCTION}

VESTA is a Monte Carlo depletion interface code developed by IRSN. From its inception, VESTA is intended to be a "generic" interface code so that it will ultimately be capable of using any Monte Carlo code or depletion module and that can be tailored to the user's needs. VESTA 2.1.5 [1] allowed for the use of any version of MCNP(X) [2] with ORIGEN 2.2 [3] or PHOENIX [1] depletion module, developed by IRSN. A new version of the code, VESTA 2.2.0, has been recently finalized. This new version introduces several new features and improvements, like the output of the secondary particles spectra and the integration of the MORET 5 [4] Monte-Carlo code.

Depletion codes such as VESTA are widely used to calculate the evolution of a material subjected to radiation (be it neutrons or another type of particle) for a wide variety of applications in the fields of nuclear safety, radiation protection and environmental health safety. Other important applications for this type of codes include fuel cycle studies, criticality safety, nuclear safeguards, waste characterization, etc. For application in these fields, experimental validation is paramount.

VESTA 2.1.5 has been validated for an isotopic inventory (of 65 isotopes) from 41 samples of radiochemical measurements and 52 measurements of residual power, using the JEFF-3.1 and ENDF/BVII.0 nuclear data libraries [5, 6, 7]. It contains a wide variety of reactors (PWR, BWR, VVER) and fuel (UOX, MOX) types with and without burnable absorbers. It is validated using MCNPX-2.6.0 as the transport module and PHOENIX as the depletion module. For the experimental validation of VESTA 2.2.0, new samples with radiochemical analysis are being added, namely JAERI BWR $\mathrm{UO}_{2}-\mathrm{Gd}_{2} \mathrm{O}_{3}$ fuel samples and REGAL PWR $\mathrm{UO}_{2}-\mathrm{Gd}_{2} \mathrm{O}_{3}$ fuel samples measured at low burn up. VESTA 2.2.0 
experimental validation, using the JEFF-3.2 and ENDF/B-VII.1 nuclear data libraries, is ongoing. It will be based basically on several fuel samples used for VESTA 2.1.5 validation, in particular the GU3 sample of the ARIANE program [8] from the SFCOMPO database. For this paper, we will limit ourselves to this GU3 sample extracted from a PWR $\mathrm{UO}_{2}$ assembly.

\section{VESTA simulations}

\subsection{The VESTA code}

VESTA is a code developed by IRSN, which couples a Monte Carlo neutron transport code with a depletion module. In VESTA, both the Boltzmann and Bateman equations are coupled and form a closed and complete system of equations that describes the transport of particles as a function of time. The basic tasks of VESTA can be summarized as follows for every step in the evolution calculation:

- Perform a steady state transport calculation and update the spectral and spatial averaged one group reaction rates, cross sections (for every possible nuclide and reaction in the transmutation chains), flux values - and other relevant data for use by the depletion module;

- Solve the Bateman equations for every burn up zone in the problem using the data derived by the transport calculation;

- Pass on the new material composition for the next time step.

VESTA allows the depletion of materials for different geometrical scales, and the calculation of the material isotopic compositions during irradiation at the fuel rod level. VESTA 2.2.0 allows for the use of either MCNP(X/6) or MORET 5 transport codes, and both ORIGEN 2.2, PHOENIX, or FISPACT [9] depletion modules. MCNP6 is used by default when choosing MCNP transport module with VESTA 2.2.0 version. We will limit ourselves to the use of MCNP6 and PHOENIX for the validation case presented in this paper.

\subsection{Description of the simulation}

The Actinide Research In A Nuclear Element (ARIANE) program [8] examined irradiated MOX (Mixed OXide) and LEU (Low Enriched Uranium) fuel samples in both commercial PWR (Pressurized Water Reactor) and BWR (Boiling Water Reactors) power reactors. The ARIANE.GU3 sample is a PWR UO 2

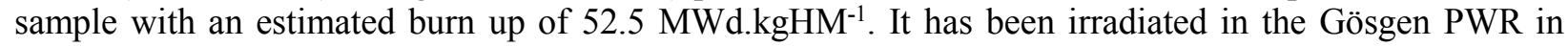
Switzerland between 1994 and 1997 during three cycles. An overview of the sample characteristics is given in Table I. The fuel rod containing the GU3 sample was extracted from the original assembly after two cycles and inserted into another assembly for the third and final cycle. After $\sim 2$ years of cooling time, the $10 \mathrm{~cm}$ sample was cut from the middle of the rod, between 122.42 and $132.42 \mathrm{~cm}$ from the fuel bottom. It was analyzed by two of the laboratories involved in the ARIANE program, being ITU (Institute for Transuranium Elements, Germany) and SCK-CEN (Belgian Nuclear Research Center, Belgium). One eighth of the $15 \times 15$ fuel assembly containing the fuel sample has been modeled in 2D using VESTA 2.2.0. Every pin is considered separately (without any radial zones) for a total of 32 zones as illustrated in Figure 1. The surrounding assemblies were not included in the model (but their effect is simulated by applying optical reflections around the 3 surfaces). The rod position change (from F7 to G5) between the second and third irradiation cycle has been modeled explicitly. All the other fuel materials were replaced by new compositions. The water moderator in this model contains an average boron concentration of 650 $\mathrm{ppm}$. The moderator and fuel pin cladding temperatures are set to $600 \mathrm{~K}$ and the fuel temperature at 900 $\mathrm{K}$. No temperature evolution as a function of time has been modeled. The power history of the assembly is deduced from the power seen by the sample specified in the experimental report. This irradiation

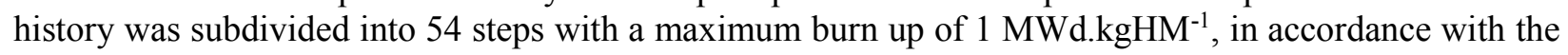
procedure laid out in [5]. 


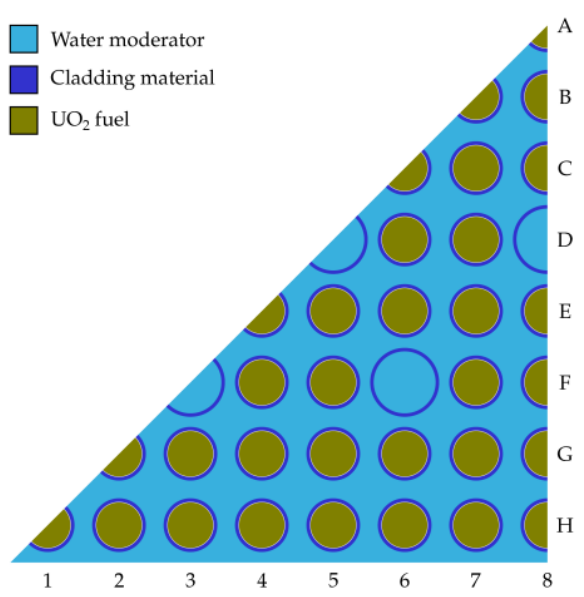

Figure 1. Assembly model for the ARIANE-GU3 sample.

\begin{tabular}{|c|c|c|}
\hline Type & PWR & \\
\hline Name & Gösgen & \\
\hline Country & Switzerland & \\
\hline Total number of assemblies & 177 & \\
\hline Fuel type & $\mathrm{UO}_{2}$ & \\
\hline${ }^{235} \mathrm{U} / \mathrm{U}$ & 4.1 & $\%$ \\
\hline $\mathrm{Pu} / \mathrm{U}+\mathrm{Pu}$ & - & $\%$ \\
\hline Gd2O3 / oxide & - & $\%$ \\
\hline Active fuel length & 355.0 & $\mathrm{~cm}$ \\
\hline Fuel density & 10.4 & g. $\mathrm{cm}^{-3}$ \\
\hline Estimated sample burn up & 52.5 & ${\mathrm{MWd} . \mathrm{kgHM}^{-1}}^{-1}$ \\
\hline Lattice type & $15 \times 15$ & \\
\hline Assembly pitch & 21.56 & $\mathrm{~cm}$ \\
\hline Pin pitch & 1.43 & $\mathrm{~cm}$ \\
\hline Fuel pins & 205 & \\
\hline Guide tubes & 20 & \\
\hline Guide tube material & Zr-4 & \\
\hline Cladding material & Zr-4 & \\
\hline
\end{tabular}

Table I. Overview of the characteristics of the ARIANE-GU3 fuel sample.

\section{VESTA VALIDATION RESULTS}

\subsection{Validation overview}

The general calculation flow when performing an experimental validation calculation using radiochemical assay data can be split up into two steps. The first step is an iterative calibration of the irradiation history using burn up tracers to accurately assess the burn up of the sample and to provide a set of reference calculated to experiment (or $\mathrm{C} / \mathrm{E}$ ) values for the measured nuclides. After determining the reference $\mathrm{C} / \mathrm{E}$ values, additional calculations have to be made to define an uncertainty range for every individual nuclide. This includes the measurement uncertainty but also the uncertainty introduced due to the irradiation history calibration. The combined value of ${ }^{145} \mathrm{Nd},{ }^{146} \mathrm{Nd}$, and ${ }^{148} \mathrm{Nd}$ burn up tracers (for each laboratory) has been applied for the normalization of the irradiation history. According to the VESTA validation procedure [5], the calibration consists of determining a global renormalization factor which is applied to the irradiation history as a whole. This renormalization constant is determined through iteration until the relative difference between the combined $\mathrm{C} / \mathrm{E}$ value for the burn up tracers to the target $\mathrm{C} / \mathrm{E}$ value (being 1.000 in this case) is less than $0.1 \%$. The power history used for the calculation of this sample is based on the sample power from which VESTA derives the assembly power distribution.

It should also be expressed that ${ }^{238} U$ composition results are not significant because ${ }^{238} U$ is the reference nuclide for the experimental validation of VESTA, by which all the composition results are normalized to get rid of unit issues, as explained in [5].

\subsection{Validation results using ENDF/B-VII.1 and JEFF-3.2 libraries}

The result of the calibration and the estimation of the burn up range for both analyses is given in Table II for both ENDF/B-VII.1 and JEFF-3.2 libraries. The burn up estimates of the sample based on the combined value of $\mathrm{Nd}$ isotopes are respectively of the order of 6-7 \% and $0.5-1 \%$ below the reported burn

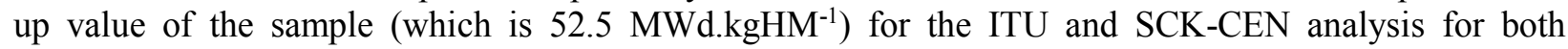
libraries. The final error on the burn up value is about $1.5 \%$ at $3 \sigma$ for the SCK analysis, while $5.5 \%$ 
uncertainty is obtained for the ITU analysis for the 2 libraries. This leads to a total uncertainty on the burn

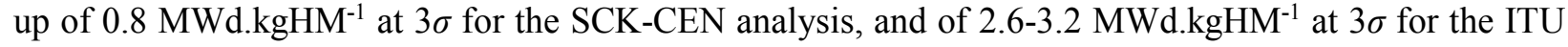
analysis, due to the burn up level of the sample. The reported experimental burn up value is therefore correctly within the uncertainty range on the calculated burn up value for the SCK-CEN analysis and just outside the upper limit of the uncertainty range for the ITU analysis. This might indicate a possible measurement issue with the ITU Nd data.

Table II. Scaling factors and burn up range estimates of the ARIANE GU3 sample using the ENDF/B-VII.1 and JEFF-3.2 libraries.

\begin{tabular}{|c|c|c|c|c|c|}
\hline & Laboratory & \multicolumn{2}{|c|}{ ITU } & \multicolumn{2}{|c|}{ SCK-CEN } \\
\hline \multirow{5}{*}{$\begin{array}{l}\text { ENDF/ } \\
\text { B-VII.1 }\end{array}$} & & $\begin{array}{l}\text { Normalization } \\
\text { factor }\end{array}$ & 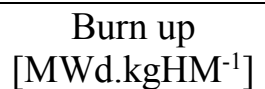 & $\begin{array}{l}\text { Normalization } \\
\text { factor }\end{array}$ & 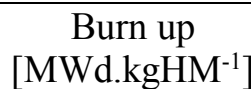 \\
\hline & $\mathrm{Nd}$ reference & 0.9753 & 49.47 & 1.0300 & 52.24 \\
\hline & $\mathrm{Nd}$ lower limit & 0.9222 & 46.78 & 1.0142 & 51.44 \\
\hline & Nd upper limit & 1.0285 & 52.17 & 1.0457 & 53.04 \\
\hline & Laboratory & \multicolumn{2}{|c|}{ ITU } & \multicolumn{2}{|c|}{ SCK-CEN } \\
\hline \multirow{4}{*}{$\begin{array}{l}\text { JEFF- } \\
3.2\end{array}$} & & $\begin{array}{l}\text { Normalization } \\
\text { factor }\end{array}$ & 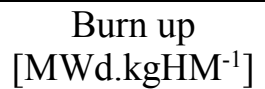 & $\begin{array}{c}\text { Normalization } \\
\text { factor }\end{array}$ & 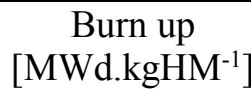 \\
\hline & $\mathrm{Nd}$ reference & 0.9703 & 49.22 & 1.0252 & 52.0 \\
\hline & Nd lower limit & 0.9174 & 46.53 & 1.0095 & 51.21 \\
\hline & Nd upper limit & 1.0231 & 51.89 & 1.0408 & 52.79 \\
\hline
\end{tabular}

Table III gives the reference VESTA 2.2.0 C/E results obtained for each measured actinide, using ENDF/B-VII.1 and JEFF-3.2 libraries, compared to the previous VESTA 2.1.5 validation results obtained for this sample with ENDF/B-VII.0 and JEFF-3.1[10]. Their associated uncertainty due to burn up is not displayed on the table, for visibility reasons, but the whole uncertainty range is displayed on Figure 2 for ${ }^{238} \mathrm{Pu}$.

The results are provided only for the SCK-CEN analysis, as the ITU results are suspected to exhibit some measurement issues as explained before. It should be noted here that the impact of using a new VESTA version, as well as a new MCNP version (MCNP6 instead of MCNPX-2.6.0) has been tested and found to be not significant, both on the flux, the $k_{\infty}$ and the isotopic concentrations, which allows to derive conclusions on impacts between libraries.

The $U$ and $\mathrm{Pu}$ contents (with the exception of ${ }^{234} \mathrm{U}$ and ${ }^{244} \mathrm{Pu}$ ) are correctly estimated within $5 \%$, both for ENDF/B-VII.1 and JEFF-3.2, as it was the case for ENDF/B-VII.0 and JEFF-3.1. The impact of using JEFF-3.2 instead of JEFF-3.1, as well as ENDF/B-VII.1 instead of ENDF/B-VII.0 is not significant for the main $\mathrm{U}$ and $\mathrm{Pu}$ isotopes, except for ${ }^{238} \mathrm{Pu}$ which shows a $\mathrm{C} / \mathrm{E}$ increase of around $11 \%$ using ENDF/BVII.1. The results, which show an underestimation with ENDF/B-VII.0, are improved with ENDF/BVII.1. The strong increase of the ${ }^{238} \mathrm{Pu} \mathrm{C} / \mathrm{E}$ results with ENDF/B-VII.1 is directly linked to the decrease of the ${ }^{238} \mathrm{Pu}$ capture cross-section in ENDF/B-VII.1 in the thermal energy range. The Am content is

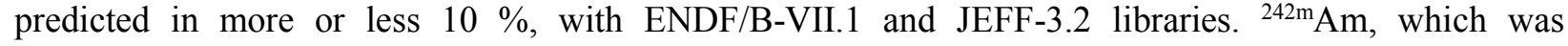
overestimated by $10 \%$ with JEFF-3.1, is decreased and show a nice agreement with JEFF-3.2. And ${ }^{243} \mathrm{Am}$, which was overestimated with ENDF/B-VII.0, is decreased by $10 \%$ and show a nice agreement with ENDF/B-VII.1. The ${ }^{243} \mathrm{Cm}$ content is strongly increased (by $30 \%$ ) in both ENDF/B-VII.1 and JEFF3.2, which increases the overestimation already observed with ENDF/B-VII.0 and JEFF-3.1. The ${ }^{245} \mathrm{Cm}$ result, on the other hand, is increased by $\sim 8 \%$ with JEFF-3.2, which improves the result.

The $\mathrm{C} / \mathrm{E}$ results obtained for fission products are not displayed on this paper, but the impact of the new ENDF/B-VII.1 and JEFF-3.2 libraries is not significant. The exception is ${ }^{90} \mathrm{Sr}$ using ENDF/B-VII.1 which 
is significantly decreased and becomes largely underestimated, as well as some few noticeable impacts with JEFF-3.2 for ${ }^{148} \mathrm{Sm},{ }^{149} \mathrm{Sm},{ }^{150} \mathrm{Sm}$ and ${ }^{154} \mathrm{Eu} .{ }^{148} \mathrm{Sm}$ is increased by $20 \%,{ }^{149} \mathrm{Sm}$ and ${ }^{150} \mathrm{Sm}$ are both decreased by $10 \%$ which strongly improve the $\mathrm{C} / \mathrm{E}$ result, and finally ${ }^{154} \mathrm{Eu}$ is strongly increased, which also improves the result. Figure 2 shows, as an example, the ${ }^{238} \mathrm{Pu} \mathrm{C} / \mathrm{E}$ results, and their associated burn up and measurement uncertainties in $3 \sigma$, obtained with ENDF/B-VII.1 and JEFF-3.2, compared to the ones obtained with ENDF/B-VII.0 and JEFF-3.1, for the two analyses. The results, which show an underestimation with ENDF/B-VII.0 and JEFF-3.1, are both increased and improved with ENDF/B-VII.1 and JEFF-3.2.

Table III. Reference C/E results $( \pm 1 \sigma)$ obtained with ENDF/B-VII.1 and JEFF-3.2 libraries, compared to the ones obtained with ENDF/B-VII.0 and JEFF-3.1, for the ARIANE GU3 sample and the SCK analysis.

\begin{tabular}{|c|c|c|c|c|c|c|}
\hline Isotope & $\begin{array}{c}\text { Reference C/E } \\
\text { ENDF/B-VII.0 [10] }\end{array}$ & $\begin{array}{l}\text { Reference C/E } \\
\text { ENDF/B-VII.1 }\end{array}$ & $\begin{array}{l}\text { Impact } \\
\text { ENDF }\end{array}$ & $\begin{array}{l}\text { Reference C/E } \\
\text { JEFF-3.1 [10] }\end{array}$ & $\begin{array}{c}\text { Reference C/E } \\
\text { JEFF-3.2 }\end{array}$ & $\begin{array}{l}\text { Impact } \\
\text { JEFF }\end{array}$ \\
\hline${ }^{234} \mathbf{U}$ & $1.416 \pm 0.039$ & $1.418 \pm 0.039$ & & $1.417 \pm 0.039$ & $1.436 \pm 0.039$ & \\
\hline${ }^{235} \mathbf{U}$ & $1.018 \pm 0.006$ & $1.016 \pm 0.006$ & $-0.2 \%$ & $1.033 \pm 0.006$ & $1.036 \pm 0.006$ & $0.3 \%$ \\
\hline${ }^{236} \mathbf{U}$ & $1.000 \pm 0.006$ & $0.999 \pm 0.006$ & $-0.1 \%$ & $0.992 \pm 0.006$ & $0.991 \pm 0.006$ & $-0.1 \%$ \\
\hline${ }^{238} \mathbf{U}$ & 1.000 & 1.000 & - & 1.000 & 1.000 & - \\
\hline${ }^{237} \mathbf{N p}$ & $0.892 \pm 0.036$ & $0.883 \pm 0.036$ & $-1.0 \%$ & $0.906 \pm 0.037$ & $0.893 \pm 0.036$ & $-1.4 \%$ \\
\hline${ }^{238} \mathrm{Pu}$ & $0.916 \pm 0.0$ & $1.02 \pm 0.018$ & & $0.939 \pm 0.016$ & $0.966 \pm 0$. & 2.9 \\
\hline${ }^{239} \mathrm{Pu}$ & $0.963 \pm 0.005$ & $0.959 \pm 0.005$ & & $0.945 \pm 0.005$ & $0.942 \pm 0.005$ & \\
\hline${ }^{240} \mathrm{Pu}$ & $0.944 \pm 0.005$ & $0.948 \pm 0.005$ & $0.4 \%$ & $0.966 \pm 0.005$ & $0.963 \pm 0.005$ & $-0.3 \%$ \\
\hline${ }^{241} \mathrm{Pu}$ & $008 \pm 0.005$ & $1.009 \pm 0.005$ & & $0.999 \pm 0.005$ & $0.999 \pm 0.005$ & \\
\hline${ }^{242} \mathrm{Pu}$ & $0.978 \pm 0.005$ & $1.014 \pm 0.005$ & $3.7^{\circ}$ & $1.009 \pm 0.005$ & $1.009 \pm 0.005$ & $0.0 \%$ \\
\hline${ }^{244} \mathrm{Pu}$ & $0.587 \pm 0.147$ & $0.575 \pm 0.144$ & $-2.0 \%$ & $0.578 \pm 0.145$ & $0.575 \pm 0.144$ & $-0.5 \%$ \\
\hline${ }^{241} \mathrm{Am}$ & $1.114 \pm 0.022$ & $1.091 \pm 0.022$ & & $1.074 \pm 0.021$ & $1.058 \pm 0.021$ & \\
\hline${ }^{242 m} \mathrm{Am}$ & $0.853 \pm 0.047$ & $0.886 \pm 0.049$ & $3.9 \%$ & $1.091 \pm 0.060$ & $1.012 \pm 0.056$ & $-7.2 \%$ \\
\hline${ }^{243} \mathrm{Am}$ & $1.177 \pm 0.023$ & $1.058 \pm 0.021$ & & $1.104 \pm 0.022$ & $1.101 \pm 0.022$ & $-0.3 \%$ \\
\hline${ }^{242} \mathrm{Cm}$ & $0.886 \pm 0.020$ & $0.927 \pm 0.021$ & $4.6 \%$ & $0.902 \pm 0.020$ & $0.94 \pm 0.021$ & $4.2 \%$ \\
\hline${ }^{243} \mathrm{Cm}$ & $1.135 \pm 0.111$ & $1.46 \pm 0.143$ & $28.6 \%$ & $1.132 \pm 0.111$ & $1.489 \pm 0.146$ & \\
\hline${ }^{244} \mathrm{Cm}$ & $0.932 \pm 0.017$ & $0.945 \pm 0.017$ & $1.4 \%$ & $0.862 \pm 0.016$ & $0.854 \pm 0.016$ & $-0.9 \%$ \\
\hline${ }^{245} \mathrm{Cm}$ & $1.047 \pm 0.029$ & $1.047 \pm 0.029$ & & $0.875 \pm 0.025$ & $0.943 \pm 0.026$ & $7.8 \%$ \\
\hline${ }^{246} \mathrm{Cm}$ & $0.89 \pm 0.048$ & $0.838 \pm 0.045$ & $-5.8 \%$ & $0.723 \pm 0.039$ & $0.737 \pm 0.039$ & $1.9 \%$ \\
\hline
\end{tabular}

\subsection{Validation results using ENDF/B-VIII.0 and JEFF-3.3 libraries}

Comparisons of the multiplication factor values obtained for different ENDF/B and JEFF libraries as a function of irradiation time are shown on Figure 3. On the top, the $k_{\infty}$ results obtained with VESTA 2.2.0 for ENDF/B-VIII.0, ENDF/B-VII.1, ENDF/B-VII.0, JEFF-3.3, JEFF-3.2 and JEFF-3.1 nuclear data libraries are displayed for the ARIANE-GU3 fuel sample. The error bars are not visible due to the small statistical errors being less than $35 \mathrm{pcm}$ in $3 \sigma$ on the VESTA calculations. On the bottom, the difference for each library with respect to ENDF/B-VIII.0 in pcm is displayed (with its combined statistical uncertainty in $3 \sigma$ ). 

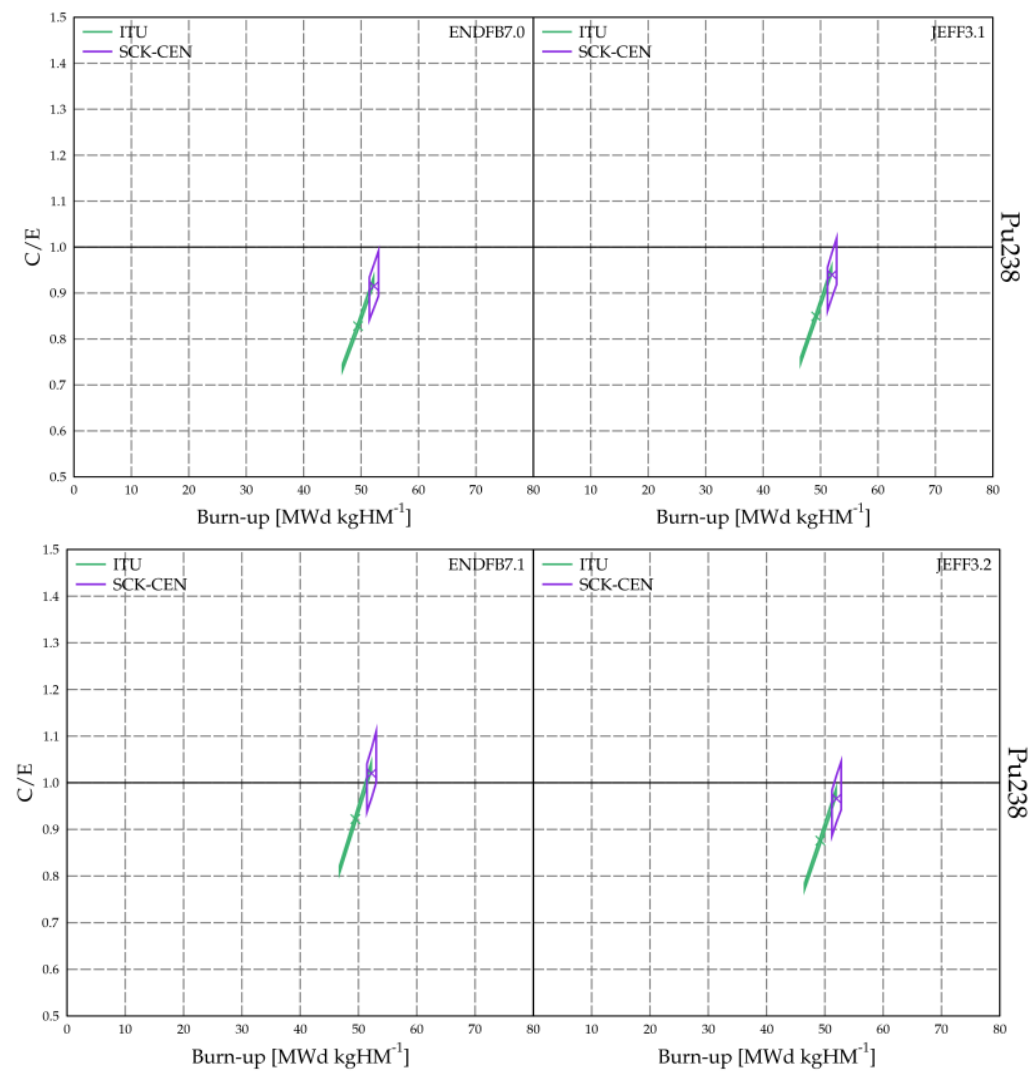

Figure 2. ${ }^{238} \mathrm{Pu} \mathrm{C} / \mathrm{E}$ results obtained with ENDF/B-VII.1 and JEFF-3.2 libraries, compared to the ones obtained with ENDF/B-VII.0 and JEFF-3.1 for the ARIANE-GU3 sample.

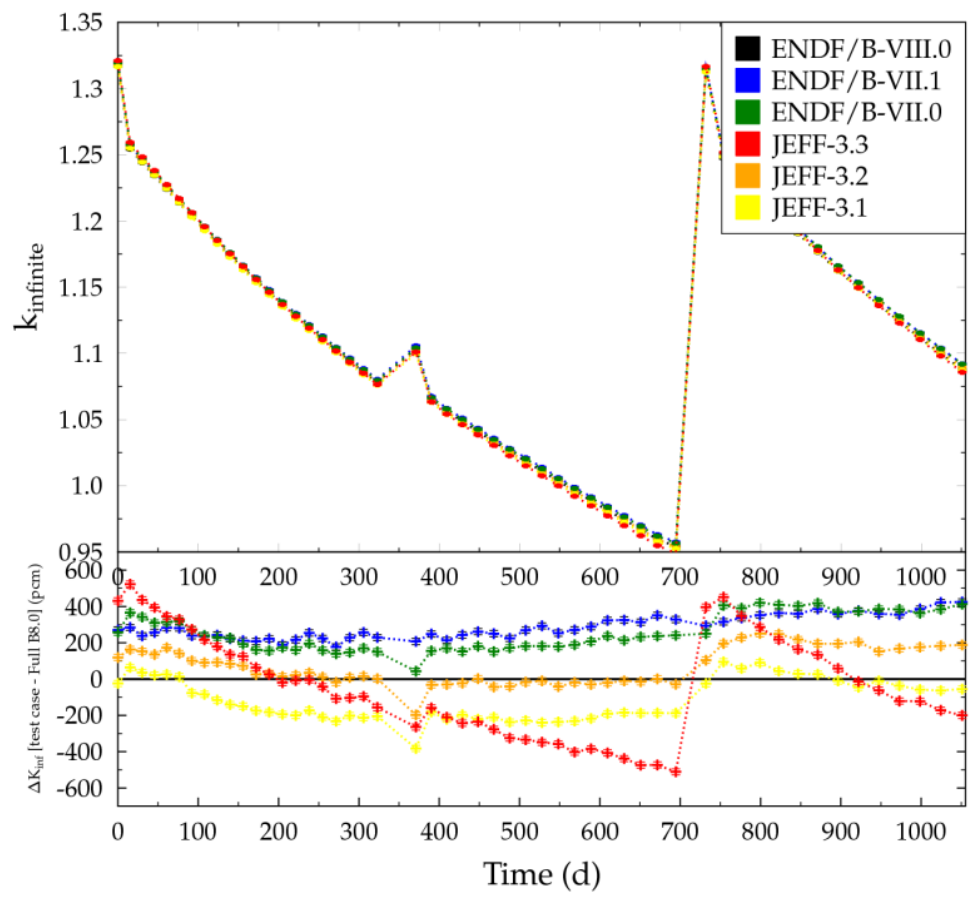

Figure 3. $k_{\infty}$ VESTA results obtained for the different nuclear data libraries for the ARIANEGU3 sample. 
The $k_{\infty}$ results obtained with the five libraries, leaving JEFF-3.3 aside firstly, show some discrepancies with irradiation time, up to $800 \mathrm{pcm}$ (between ENDF/B-VII.0 and JEFF-3.1), but the $\Delta \mathrm{k}_{\text {inf }}$ with respect to the ENDF/B-VIII.0 result stays more or less constant. Only a small impact of the $2^{\text {nd }}$ cooling time and change of position of the sample (at 700 - 750 days) can be noticed on the libraries discrepancies on $\mathrm{k}_{\text {inf }}$ results. The JEFF-3.3 results, on the other hand, show a huge burn up effect, and are very surprising. JEFF-3.3 $k_{\infty}$ results are overestimating ENDF/B-VIII.0 ones by $\sim 500 \mathrm{pcm}$ at the beginning of the first cycle, whereas at the end of the $2^{\text {nd }}$ cycle they are underestimating ENDF/B-VIII.0 by $\sim 500 \mathrm{pcm}$. The JEFF-3.3 $k_{\infty}$ decreases so much with burn up that a huge jump, of around $1000 \mathrm{pcm}$, is observed during the rod change of assembly at $3^{\text {rd }}$ cycle. More investigations are needed to understand this phenomena. Fissile isotopes, especially ${ }^{239} \mathrm{Pu}$, whose capture cross section was increased by $2 \%$ in JEFF-3.3 (compared to JEFF-3.2), might be good candidates.

Validation calculations were also performed using ENDF/B-VIII.0 and JEFF-3.3 libraries for the ARIANE-GU3 sample. The final error on the burn up value is about $1.5 \%$ at $3 \sigma$ for the SCK-CEN analysis and about $5.5 \%$ uncertainty is obtained for the ITU analysis for both libraries, which does not differ from the values obtained with ENDF/B-VII.1 and JEFF-3.2. Table IV gives the reference VESTA 2.2.0 C/E results obtained for each measured actinide, using ENDF/B-VIII.0 and JEFF-3.3 libraries, compared to the ENDF/B-VII.1 and JEFF-3.2 results. The actinides contents are globally not much impacted by the use of the new libraries.

Table IV. Reference C/E results obtained with ENDF/B-VIII.0 and JEFF-3.3 libraries, compared to the ones obtained with ENDF/B-VII.1 and JEFF-3.2, for the ARIANE GU3 sample and the SCK analysis.

\begin{tabular}{|c|c|c|c|c|}
\hline Isotope & $\begin{array}{l}\text { Reference C/E } \\
\text { ENDF/B-VIII.0 }\end{array}$ & \begin{tabular}{|c|} 
Impact \\
previous \\
ENDF \\
\end{tabular} & $\begin{array}{c}\text { Reference C/E } \\
\text { JEFF-3.3 }\end{array}$ & $\begin{array}{c}\text { Impact } \\
\text { previous } \\
\text { JEFF }\end{array}$ \\
\hline${ }^{234} \mathbf{U}$ & $1,408 \pm 0.038$ & $-0,7 \%$ & $1,431 \pm 0.038$ & $-0,3 \%$ \\
\hline${ }^{235} \mathbf{U}$ & $0,981 \pm 0.005$ & $-3,4 \%$ & $1,004 \pm 0.006$ & $-3,1 \%$ \\
\hline${ }^{236} \mathbf{U}$ & $1,014 \pm 0.006$ & $1,5 \%$ & $0,994 \pm 0.006$ & $0,3 \%$ \\
\hline${ }^{2338} \mathbf{U}$ & 1 & - & 1 & - \\
\hline${ }^{237} \mathbf{N p}$ & $0,895 \pm 0.036$ & $1,4 \%$ & $0,904 \pm 0.036$ & $1,2 \%$ \\
\hline${ }^{238} \mathrm{Pu}$ & $1,032 \pm 0.018$ & $1,2 \%$ & $1,045 \pm 0.018$ & $8,2 \%$ \\
\hline${ }^{239} \mathrm{Pu}$ & $0,96 \pm 0.005$ & $0,1 \%$ & $0,93 \pm 0.005$ & $-1,3 \%$ \\
\hline${ }^{240} \mathrm{Pu}$ & $0,955 \pm 0.005$ & $0,7 \%$ & $0,968 \pm 0.005$ & $0,5 \%$ \\
\hline${ }^{241} \mathrm{Pu}$ & $1,005 \pm 0.005$ & $-0,4 \%$ & $0,999 \pm 0.005$ & $0,0 \%$ \\
\hline${ }^{242} \mathrm{Pu}$ & $1,004 \pm 0.005$ & $-1,0 \%$ & $1,008 \pm 0.005$ & $-0,1 \%$ \\
\hline${ }^{244} \mathrm{Pu}$ & $0,605 \pm 0.152$ & $5,2 \%$ & $0,564 \pm 0.141$ & $-1,9 \%$ \\
\hline${ }^{241} \mathrm{Am}$ & $1,086 \pm 0.021$ & $-0,5 \%$ & $1,058 \pm 0.021$ & $0,0 \%$ \\
\hline${ }^{242 \mathrm{~m}} \mathrm{Am}$ & $0,88 \pm 0.049$ & $-0,7 \%$ & $1,008 \pm 0.056$ & $-0,4 \%$ \\
\hline${ }^{243} \mathrm{Am}$ & $1,046 \pm 0.021$ & $-1,1 \%$ & $1,051 \pm 0.021$ & $-4,5 \%$ \\
\hline${ }^{242} \mathrm{Cm}$ & $0,921 \pm 0.021$ & $-0,6 \%$ & $0,938 \pm 0.021$ & $-0,2 \%$ \\
\hline${ }^{243} \mathrm{Cm}$ & $1,454 \pm 0.142$ & $-0,4 \%$ & $1,485 \pm 0.145$ & $-0,3 \%$ \\
\hline${ }^{244} \mathrm{Cm}$ & $0,939 \pm 0.017$ & $-0,6 \%$ & $0,928 \pm 0.017$ & $8,7 \%$ \\
\hline${ }^{245} \mathrm{Cm}$ & $1,051 \pm 0.029$ & $0,4 \%$ & $1,033 \pm 0.029$ & $9,5 \%$ \\
\hline${ }^{246} \mathrm{Cm}$ & $0,847 \pm 0.045$ & $1,1 \%$ & $0,809 \pm 0.043$ & $9,8 \%$ \\
\hline
\end{tabular}


The only exceptions are: ${ }^{235} \mathrm{U}$, which is decreased by $3 \%$ for both cases but is still in a good agreement with the experiment; ${ }^{238} \mathrm{Pu}$, which is increased by $8 \%$ in JEFF-3.3 and also stays in a good agreement; ${ }^{244} \mathrm{Pu}$, which is increased by $5 \%$ in ENDF/B-VIII. $0 ;{ }^{243} \mathrm{Am}$, which is decreased by $5 \%$ in JEFF-3.3 and becomes in a good agreement, and finally ${ }^{244} \mathrm{Cm},{ }^{245} \mathrm{Cm}$ and ${ }^{246} \mathrm{Cm}$ which are increased by almost $10 \%$ in JEFF-3.3, which improve the results. The impact on the $\mathrm{C} / \mathrm{E}$ results obtained for fission products of the new ENDF/B-VIII.0 instead of ENDF/B-VII.1 is not significant. The exception is ${ }^{90} \mathrm{Sr}$, which is strongly increased. For JEFF-3.3, on the other hand, a significant amount of fission products are impacted, among which europium isotopes, ${ }^{155} \mathrm{Gd},{ }^{109} \mathrm{Ag},{ }^{125} \mathrm{Sb},{ }^{129} \mathrm{I}$ and ${ }^{125} \mathrm{Cs}$. However, due to their large measurement uncertainty, it remains difficult to conclude.

\section{CONCLUSIONS}

The validation of the VESTA 2.2.0 Monte Carlo depletion code has been initiated using the ARIANEGU3 chemical assay data from the OECD/NEA SFCOMPO database. The chemical analyses of the studied fuel sample were performed by 2 independent laboratories at the end of irradiation and cooling time. US and European evaluated nuclear data libraries, namely ENDF/B-VII.1 and JEFF-3.2, but also the more recent ENDF/B-VIII.0 and JEFF-3.3 were used for the VESTA 2.2.0 calculations. The C/E results were compared to the previous VESTA 2.1.5 validation results obtained using ENDF/B-VII.0 and JEFF3.1 nuclear data libraries. The $\mathrm{U}$ and $\mathrm{Pu}$ contents are globally correctly estimated within $5 \%$, both for ENDF/B-VII.1 and JEFF-3.2, as it was the case for ENDF/B-VII.0 and JEFF-3.1. The ${ }^{238} \mathrm{Pu}$ results, which show an underestimation with ENDF/B-VII.0, are nicely improved with ENDF/B-VII.1. Am and Cm isotopes are also mainly improved. They tend to be unchanged or even more improved using the new JEFF-3.3 and ENDF/B-VIII.0 libraries, despite the fact that the JEFF-3.3 $\mathrm{k}_{\text {inf }}$ results are showing a huge burn up effect.

\section{ACKNOWLEDGMENTS}

The authors want to thank Q. Barreau for the VESTA 2.2.0 validation calculations.

\section{REFERENCES}

1. W. Haeck, B. Cochet, L. Aguiar, "Monte Carlo depletion calculations using VESTA 2.1 new features and perspectives," Proceedings of PHYSOR 2012, Knoxville, USA (2012).

2. J. S. Hendricks et al., "MCNPX, VERSION 2.6.a", LA-UR-05-8225, Los Alamos National Laboratory, USA (2005).

3. A. G. Croff, "ORIGEN2 - A Revised and Updated Version of the Oak Ridge Isotope Generation and Depletion Code", ORNL-5621, Oak Ridge National Laboratory, USA (1980).

4. B. Cochet, A. Jinaphanh, L. Heulers, O. Jacquet "Capabilities overview of the MORET 5 Monte Carlo code", Annals of Nuclear Energy 82 (2015) 74-84.

5. W. Haeck, "VESTA - General procedure for experimental validation of depletion codes", IRSN Report PSN-EXP/SNC/2012-266, January 2013.

6. W. Haeck, B. Cochet, F. Bernard, "Experimental validation of VESTA 2.1," Proceedings of SNA+MC 2013, Paris, October 27-31, 2013.

7. W. Haeck, "Experimental validation of VESTA 2.1.5 Volume 1: Methodology and overview of results", IRSN Report PSN-EXP/SNC/2014-237, September 2014.

8. ARIANE "ARIANE International Program Final Report", Oak Ridge National Laboratory, ORNL/SUB/97-XSV750-1, May 1, 2003

9. "FISPACT II: An Advanced Simulation System for Activation, Transmutation and Material Modelling," Nuclear Data Sheets 139 (2017) 77-137.

10. R. Ichou, "Experimental validation of VESTA 2.1.5 Volume 2: Radiochemical assay data - ARIANE program", IRSN Report PSN-EXP/SNC/2014-238, September 2014. 